\title{
Genomic Sequence and Analysis of EhV-99B1, a New Coccolithovirus from the Norwegian Fjords
}

\author{
A. Pagarete $^{a} \quad$ A. Lanzén ${ }^{a, b} \quad$ P. Puntervoll ${ }^{b} \quad$ R.A. Sandaa ${ }^{a} \quad$ A. Larsen ${ }^{c}$ \\ J.B. Larsen ${ }^{\mathrm{a}}$ M.J. Allen ${ }^{\mathrm{d}}$ G. Bratbak ${ }^{\mathrm{a}}$ \\ a Department of Biology, University of Bergen, ${ }^{b}$ Computational Biology Unit, Uni Computing, Uni Research,

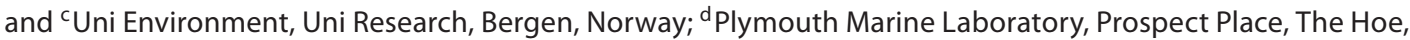 \\ Plymouth, UK
}

\section{Key Words}

Coccolithovirus - Emiliania huxleyi virus - Marine virus • Giant virus $\cdot$ Selfish genetic element $\cdot$ Horizontal gene transfer

\begin{abstract}
Coccolithoviruses are giant dsDNA viruses that infect Emiliania huxleyi, the most ubiquitous marine microalga. Here, we present the genome of the latest coccolithovirus strain to be sequenced, EhV-99B1, and compare it with two other coccolithovirus genomes (EhV-86 and EhV-163). EhV-99B1 shares a pairwise nucleotide identity of $98 \%$ with EhV-163 (the two strains were isolated from the same Norwegian fjord but in different years), and just $96.5 \%$ with EhV-86 (isolated in the same spring as EhV-99B1 but in the English Channel). We confirmed and extended the list of relevant genomic differences between these EhVs from the Norwegian fjord and EhVs from the English Channel, namely the removal/insertions of: a phosphate permease, an endonuclease, a transposase, and two specific tRNAs. As a whole, this study provided new clues and insights into the diversity and mechanisms driving the evolution of these large oceanic viruses, in particular those processes involving selfish genetic elements.

Copyright $\odot 2012$ S. Karger AG, Basel
\end{abstract}

Emiliania huxleyi (Haptophyta) is an abundant marine eukaryotic alga. Its carbon sequestration and calcification capacities in combination with an extensive ocean-wide distribution ensure a prime position for the species with regard to impact on global biogeochemical cycling [1]. E. huxleyi forms massive blooms in coastal and open ocean waters which are often decimated by acute lytic viral infection [2]. The action of such alga-infecting viruses is far reaching due to their profound ecological role and major impact on phytoplankton population dynamics and diversity [3]. Furthermore, the coccolithoviruses are among the largest viruses known, and have provided crucial clues to early cellular and virus evolution [4].

To date, the lytic viruses isolated that infect E. huxleyi (EhVs, genus Coccolithovirus) are all large dsDNA viruses (genome size $\sim 410 \mathrm{kbp}$, particle size $160-180 \mathrm{~nm}$ ) which belong to the nuclear-cytoplasmic large doublestranded DNA virus (NCLDV) group [5]. Due to the highly repetitive nature of the genomes, just one coccolithovirus (EhV-86) has been fully sequenced, in which 472 CDSs were identified [6], while a further eight strains (EhV-163, EhV-201, EhV-202, EhV-203, EhV-207, EhV208, EhV-84 and EhV-88) have been partially sequenced [7-11]. EhV-86 was originally isolated from the English

\section{KARGER}

Fax +4161306 1234

E-Mail karger@karger.ch

www.karger.com
(C) 2012 S. Karger AG, Basel

0300-5526/13/0561-0060\$38.00/0

Accessible online at:

www.karger.com/int
António Pagarete

Department of Biology, University of Bergen

Thormøhlensgate $53 \mathrm{~A} \& \mathrm{~B}$

NO-5006 Bergen (Norway)

Tel. +47 555844 58, E-Mail antonio.pagarete@ bio.uib.no 
Table 1. Viral isolates used in this study

\begin{tabular}{llllll}
\hline Viral isolate & Isolation date & Geographical origin & $\begin{array}{l}\text { Geographical } \\
\text { coordinates }\end{array}$ & Isolate reference & Genome reference \\
\hline EhV-86 & July 1999 & English Channel & $\begin{array}{l}50.3^{\circ} \mathrm{N}, 4.2^{\circ} \mathrm{W} \\
60.2^{\circ} \mathrm{N}, 5.2^{\circ} \mathrm{E}\end{array}$ & $\begin{array}{l}\text { Schroeder et al. [12] } \\
\text { Castberg et al. [24] }\end{array}$ & $\begin{array}{l}\text { Wilson et al. [6] } \\
\text { this study }\end{array}$ \\
EhV-163 & June 1999 & Raunfjorden, Norway & Castberg et al. [24] & Allen et al. [5] \\
\hline
\end{tabular}

Channel in 1999, the same year as EhV-99B1, while EhV163 was isolated from the same Norwegian fjord as EhV99B1 a year later in 2000 [12]. In a previous comparison of the EhV-86 and EhV-163 genomes, Allen et al. [11] reported that the contigs from EhV-163 were $95-100 \%$ identical to the EhV-86 sequence, with 18 and $10 \%$ of the available CDSs being 100\% identical on the amino acid and nucleotide levels, respectively. Unfortunately, this study was hindered by a lack of coverage: only $80 \%$ of the total EhV-163 genome could be assembled, and just $40 \%$ of the total predicted CDSs were fully sequenced and available for full analysis [11]. Despite the limited coverage, of particular note, a potential gene replacement was identified involving a putative phosphate permease and an endonuclease. Other genomic differences reported between EhV-86 and EhV-163 genomes included point mutations, in-frame insertions/deletions, and insertions/deletions leading to truncated/extended proteins [11].

In this study, we present the genome sequence of the latest coccolithovirus strain to be sequenced EhV-99B1, and compare its specific features with those of two closely related viruses, EhV-86 the fully sequenced coccolithovirus isolated in the same year as EhV-99B1, and EhV-163, the coccolithovirus isolated from the same location, but a year later (table 1).

The EhV-99B1 genome was sequenced and assembled into 16 contigs that cover approximately $98 \%$ of the genome of EhV-86. Contigs were deposited in DDBJ/EMBL/ Genbank with accession number FN429076 (full details regarding the procedures employed in this study can be found online in the supplementary file 1, www. karger.com/doi/10.1159/000341611). 434 CDSs were identified, of which 416 have homologous sequences in EhV86 (fig. 1). From these common CDSs, 70 showed 100\% conservation at the amino acid level, while 38 were also fully conserved at the nucleotide sequence level (for detailed annotations and genomic similarity data, refer to online supplementary file 2). All 26 of the NCLDV 'core genes' previously identified in EhV-86 [enumerated in 11, 13] are also present in the EhV-99B1 genome.

EhV-99B1, New Giant Virus Genome Analysis
EhV-99B1 revealed higher average nucleotide pairwise identity with EhV-163 $(98.9 \%, \sigma=3.4 \%)$ than with EhV$86(96.4 \%, \sigma=7.9 \%)$. The two viral genomes from the Norwegian fjord (EhV-99B1 and EhV-163) share all 384 CDSs previously identified in EhV-163 (from which 202 were fully sequenced, and 182 partially sequenced). The differences identified between these two viruses related to point mutations and partial insertion/deletion events (table 2). On the other hand, 35 insertion/deletion events could be identified between EhV-99B1 and EhV-86 (fig. 1; table 2), involving CDSs whose protein products are predicted to be associated with activities such as endonuclease, phosphate permease and transposase activities, as well as a $\mathrm{C}_{2} \mathrm{H}_{2}$ type zinc finger protein, DNA-binding protein and 12 putative membrane proteins. Other types of major genomic rearrangements observed were: gene duplications, intron insertions, and major internal insertions or deletions (which affect more than 30 amino acids). Nineteen CDSs have been truncated, but leaving more than $50 \%$ of valid coding sequence remaining (full details can be found in online supplementary file 2).

A particularly remarkable feature of the previously reported EhV-86 genome is that it encodes 5 tRNA genes: Leu, Arg, Gln, Asn, and Ile [6]. We now found that the EhV-99B1 genome also encodes 5 tRNAs, but the tRNA gene for leucine was replaced by another tRNA gene with the lysine anti-codon. tRNA encoding genes have also been found in other large viral genomes, namely in mimiviruses and myoviruses $[14,15]$. Since one of the most important translational optimization mechanisms is the correlation between the cellular levels of tRNA molecules and the frequencies of their corresponding codons $[16,17]$, Enav et al. [18] put forward the hypothesis that encoding its own tRNAs will increase the replication fitness of a virus. In that sense, the presence of different tRNAs in these two EhV genomes could either be directly linked to the codon usage inherent to each viral genome, or, if they have different host ranges, this could be due to different codon-selective pressures imposed by the translational biases of their respective hosts. Having no

Intervirology 2013;56:60-66 
Fig. 1. Circular representation of the 16 EhV-99B1 genome contigs, and comparison with the EhV-86 genome. Black lines in circle 1 (from outside in) indicate the position of gene deletions compared to EhV-86. Circles 2 and 3 show EhV99B1 coding sequences (forward and reverse strand, respectively). Most CDSs have homologues in EhV-86 (shown in blue); the 18 CDSs that have no counterpart in EhV86 are shown in orange. The amino acid pairwise identities between the EhV-99B1 and EhV-86 homologs are plotted as grey dots in the innermost grey circles (scale $0-100 \%$ from inside out). $100 \%$ identical genes are plotted as red dots. The genome of EhV-163 is not plotted for ease of visualization and comparison.

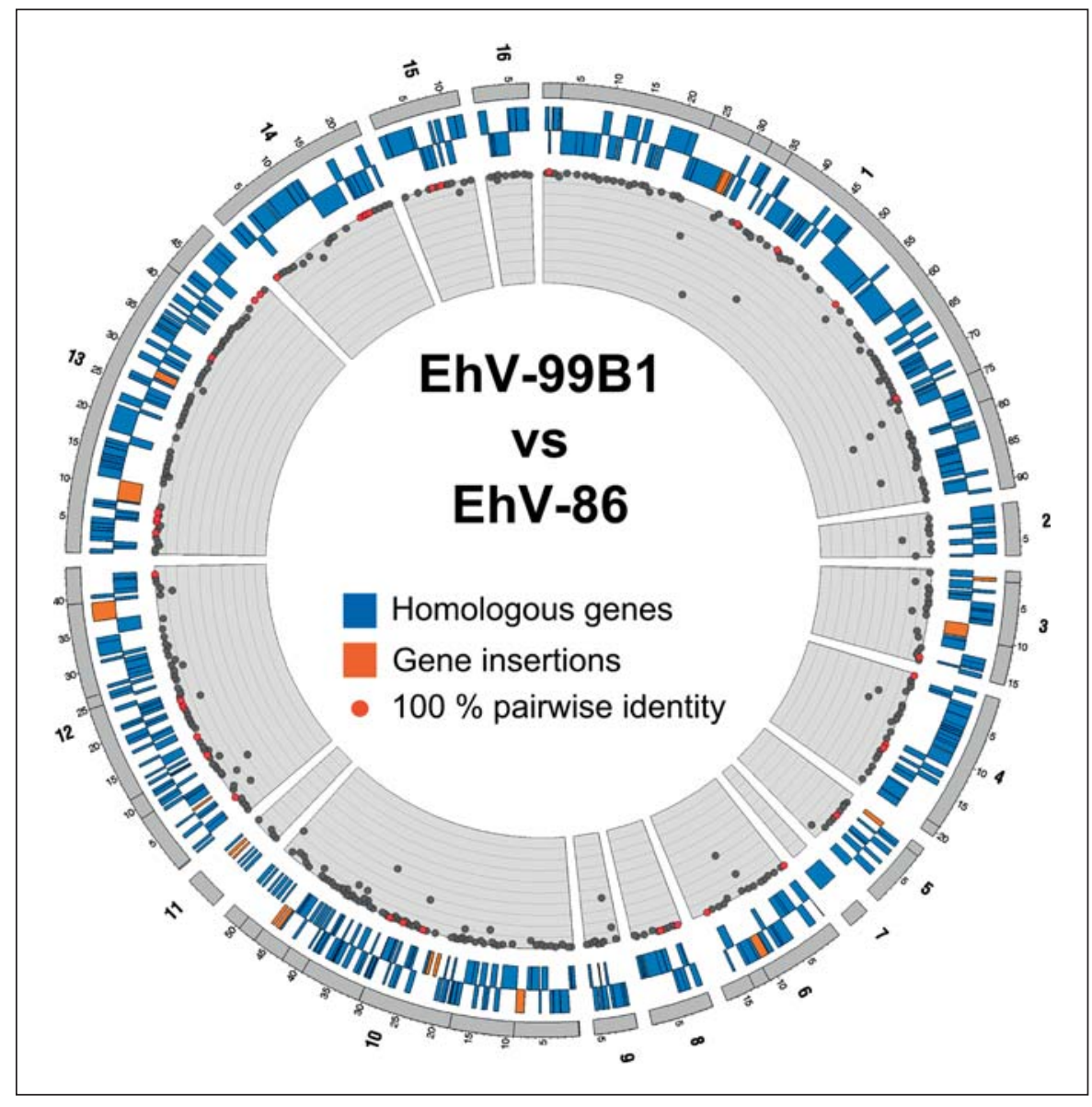

means to test the latter, due to absence of host genomic data, we focused on the codon usage for the two EhV genomes and compared those values with the tRNA set encoded in each virus (table 3 ). The leucine codon was $1.01 \%$ more prevalent in the CDSs of EhV-99B1 than in those of EhV-86, a result which opposes the codon usage hypothesis mentioned above. On the other hand, the lysine amino acid is more prevalent in the EhV-99B1 genome by a mere $0.16 \%$. Although this would fit the codon usage hypothesis, this value is smaller than the ones found for arginine and glycine, which are encoded by the two viral genomes. Hence, our results do not support a (at least clear) relationship between tRNA encoding and respective codon usage in each EhV genome. This strengthens the hypothesis that the differences in tRNA encoding might be better explained by adaptation to different hosts.

A putative phosphate permease gene (ehv117) is absent from the EhV-99B1 genome. Its absence from the genome of the other fjord virus, EhV-163, had been previously re-
Table 2. Pairwise nucleotide identity and major genomic differences registered between the genomes of EhV-99B1 and the other two viruses EhV-86 and EhV-163

\begin{tabular}{|c|c|c|}
\hline & $\begin{array}{l}\text { EhV-86 vs. } \\
\text { EhV-99B1 }\end{array}$ & $\begin{array}{l}\text { EhV-99B1 } \\
\text { vs. EhV-163 }\end{array}$ \\
\hline Pairwise nucleotide identity, $\%$ & $96.5 \pm 7.9$ & $98.9 \pm 3.4$ \\
\hline Gene deletions/insertions & 35 & 0 \\
\hline Partial insertion/deletion leading & & \\
\hline to dysfunctional gene & 5 & 1 \\
\hline Intein insertion & 1 & 0 \\
\hline Mutation(s) in start or stop codon & 1 & 1 \\
\hline Gene duplication & 1 & 0 \\
\hline
\end{tabular}

ported, but lacking confirmation [11]. A 75-bp scar remnant of the phosphate permease gene is still present at the $3^{\prime}$ end of this genome region in both viruses indicating that the fjord EhVs did once possess the phosphate permease gene. However, ehv117 is encoded by, and retained 


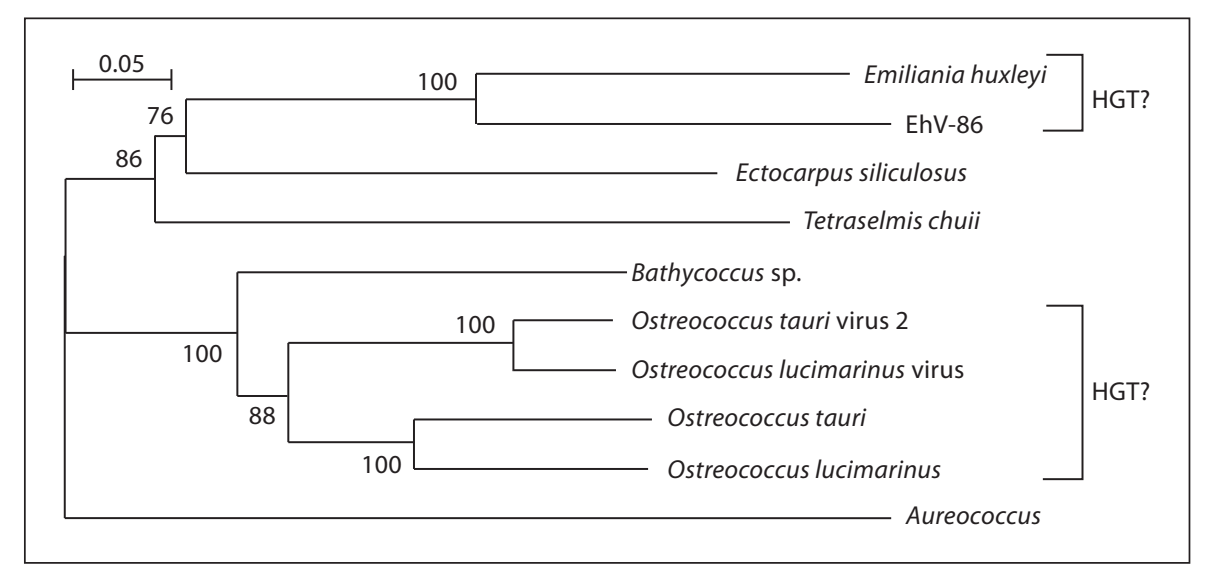

Fig. 2. Phylogenetic position of the EhV-86 phosphate permease (ehv117) among the closest homologues found in the UniProt database. The EhV gene is most closely related to a homologue gene present in E. huxleyi, its host, and may suggest HGT. A similar HGT pattern is also found in the Prasinophytes (Ostreococcus) and their viruses. UniProt accession references for presented sequences are: E. huxleyi-Q8GZT5; EhV-86 - Q4A316; Ectocarpus siliculosus - D7G0K9; Tetraselmis chuii - Q84ZX2; Bathycoccus

Table 3. Codon usage statistics for the amino acids encoded in the EhV genomes

\begin{tabular}{llll}
\hline Amino acid & \multicolumn{2}{c}{ Relative abundance } & \multirow{2}{*}{$\begin{array}{l}\text { Differ- } \\
\text { ence }\end{array}$} \\
\cline { 2 - 3 } & EhV-86 & EhV-99B1 & \\
\hline Leu (tRNA in 86) & $\mathbf{0 . 0 2 2 2 2}$ & $\mathbf{0 . 0 2 2 4 4}$ & $\mathbf{1 . 0 1}$ \\
Arg & 0.00773 & 0.00779 & 0.75 \\
Gln & 0.01757 & 0.01768 & 0.59 \\
Lys (tRNA in 99B1) & $\mathbf{0 . 0 3 5 1 5}$ & $\mathbf{0 . 0 3 5 2 1}$ & $\mathbf{0 . 1 6}$ \\
Asn & 0.01862 & 0.01865 & 0.12 \\
Ile & 0.01586 & 0.01580 & -0.40 \\
\hline
\end{tabular}

Difference stands for percent increase/decrease in codon abundance from EhV-86 to EhV-99B1. In bold are the 2 amino acids that have unique tRNAs in EhV-86 or EhV-99B1, respectively.

in, all the $9 \mathrm{EhV}$ strains isolated from the English Channel [6-10]. The involvement of the gene product in the active transport of phosphate could provide a crucial advantage for the production of new nucleotides during replication of the viral genome. Phosphate availability is one of the major constraints for productivity in marine ecosystems, and a first case of viral modulation of phosphate acquisition has recently been demonstrated for other oceanic viruses, in that case cyanophages [19]. The re- sp. - E5ESL8; OtV2 - E4WM98; Ostreococcus lucimarinus virus - E5ES24; Ostreococcus tauri - A4SA25; Ostreococcus lucimarinus - Q01EC4; Aureococcus - F0XVH5. The tree depicted here resulted from a neighbor-joining analysis performed on an amino acid alignment of the respective sequences. The evolutionary distances are in the units of the number of amino acid substitutions per site. Numbers presented on clades are percentage consensus values, calculated from 1,000 bootstrap replicates. silience of this gene in the English Channel viruses could mean that they have to face greater phosphate limitation than the EhVs from the more stable Norwegian fjord environment. However, chemical data show that the fjords are usually deprived of phosphate, in particular within the upper layers where algal blooms and infections are occurring, a condition that is a major constraint for the phytoplanktonic communities dwelling therein [20]. The explanation could nevertheless rely on a more finely structured pattern, whereby phosphate limitation is site dependent and strongly linked to the local hydrography and light conditions of the water column. A search for homologue protein sequences in the UniProt database (www.uniprot.org) reveals that this EhV phosphate permease gene is most closely related to an E. huxleyi putative phosphate permease, in what seems to be a case of horizontal gene transfer (HGT; fig. 2). Moreover, this shared E. huxleyi/EhV CDS has its closest relatives among other algae and their viruses (fig. 2), suggesting again an important role for phosphate permease in the development of planktonic viral infections. This adds to a new emerging view on how marine viruses and their cellular hosts co-evolve to face the challenges of the oceanic environment.

In both fjord viruses, in place of the putative phosphate permease, a 600-bp insert exists containing a CDS designated as ehv117A which displays a domain charac- 


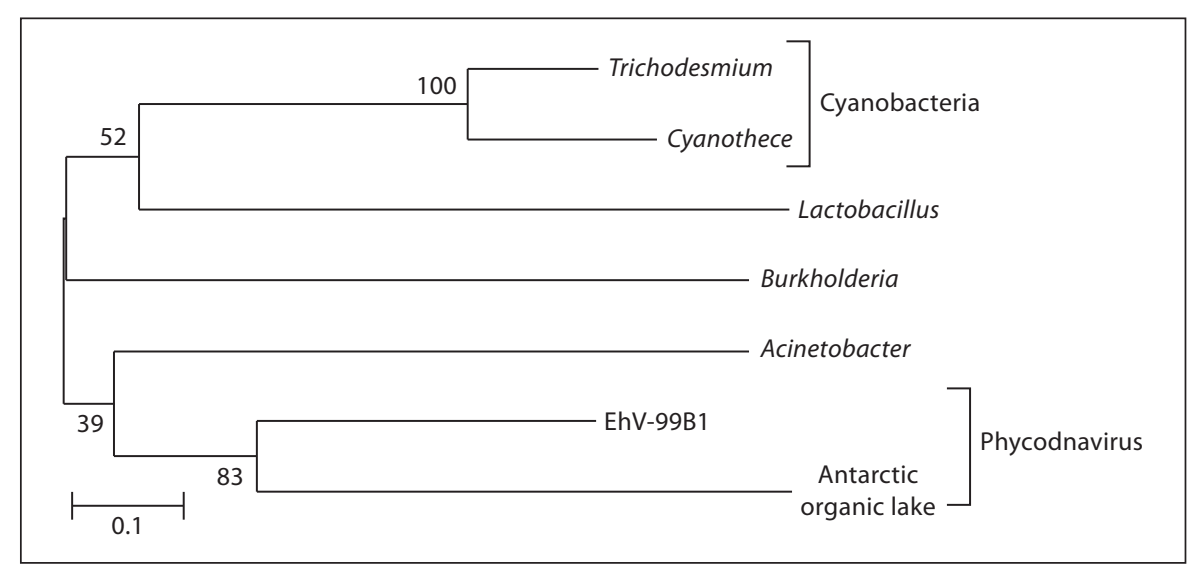

Fig. 3. Phylogenetic position of the endonuclease CDS (ehv117A) present in EhVs from the Norwegian fjord. ehv117A is most closely related to a sequence found in a metagenomic sample from an Antarctic Organic Lake. The tree depicted here resulted from a neighbor-joining analysis performed on an amino acid alignment of the respective sequences. The evolutionary distances are in the units of the number of amino acid substitutions per site. Numbers presented on clades are percentage consensus values calculated from 1,000 bootstrap replicates. UniProt accession references for presented sequences are: Tricodesmium - Q10XI5; Cyanothece E0UJ45; Lactobacillus - F0TJ73; Burkholderia - Q3JTI4; Acinetobacter - A3M519; EhV-99B1 - D2TF57; Antarctic Organic Lake - F2Y1I3. teristic of a homing endonuclease (InterPro annotation IPR002711). The direct action of this endonuclease and/ or a selfish genetic element could explain the loss of the phosphate permease gene in these fjord viruses. The endonuclease domain was also found in some bacteria and another (uncultured) phycodnavirus from an Antarctic Lake (fig. 3).

In our opinion, these two examples (ehv117 and ehv117A) represent further evidence of genetic information being horizontally transferred between and among the cellular and viral worlds. Even though the data do not allow one to say if the transfer is from the virus to the host or the host to the virus, this adds to the significance of viruses in the evolution of life $[21,22]$.

The EhV-99B1 genome also revealed the presence of a putative transposase. The locus where CDS ehv129 is found in EhV-86 is truncated with the insertion of a CDS containing two transposase motifs: an OrfB_Zn_ribbon DNA-binding domain (InterPro annotation IPR010095) and a helix-turn-helix motif (InterPro annotation IPR021027). This novel gene has been designated ehv129A. Top BLAST hits of ehv129A against UniProt refer to cyanobacteria and other large DNA viruses, Acanthamoeba polyphaga mimivirus and Paramecium bursaria chlorella virus NY2A (table 4). Although the closest sequence homology is found in mimivirus (fig. $4 \mathrm{a}$ ), the most similar gene structure is found in the P. bursaria chlorella virus NY2A (fig. 4b). No traces of this transposase were found in the EhV-86 genome, but its sequence is present in EhV-163, although previously overlooked because the CDS is split in two assembly contigs. As in the previous example, the evolutionary event that led to the removal of the gene ehv129 from these two fjord viruses is probably linked to the action of a selfish genetic element, in this case most likely the transposase itself. This increasingly prominent role of selfish genetic elements in shaping the evolution of coccolithovirus genomes is also supported by the recent discovery of an intein, encoded within the gene ehv434, with predicted self-splicing and homing endonuclease activities [23]. Curiously, this intein is also present in the Norwegian EhV strains (including EhV-99B1) and absent from their English Channel counterparts.

In conclusion, our genomic comparison analysis revealed that the novel EhV-99B1 genome is highly similar to the partial genomic sequence of another EhV isolated from the same location (EhV-163). Further, we confirmed and revealed new specific genomic traits that distinguish these two fjord EhV strains from their English Channel counterparts. Among the most remarkable features we were able to identify and/or confirm are the removal/insertion of a phosphate permease gene and endonuclease, a transposase, and two specific tRNAs. These findings point to the possible existence of an important level of 
Fig. 4. Phylogenetic position (a) and gene organization (b) of the putative transposase CDS (ehv129A) present in EhVs from the Norwegian fjord. Although its closest phylogenetic relative corresponds to a gene found in mimivirus, the most similar gene organization (composed by helix-turn-helix and OrfB domains) is found in the P. bursaria chlorella virus (PBCV) NY2A. The tree depicted here resulted from a neighbor-joining analysis performed on an amino acid alignment of the respective sequences. The evolutionary distances are in the units of the number of amino acid substitutions per site. Numbers presented on clades are percentage consensus values calculated from 1,000 bootstrap replicates. Bootstrap values (1,000 replicates) are indicated at nodes. UniProt accession references for presented sequences are: Arthrospira D4ZZT1; Synechococcus - Q2JQ93; Lyngbya - F4XNN2; Microcystis - A8YJK5; PBCV - A7RAM2; Feldmannia virus B5LW91; EhV-99B1 - D2TF67; mimivirus - Q5UPI0.
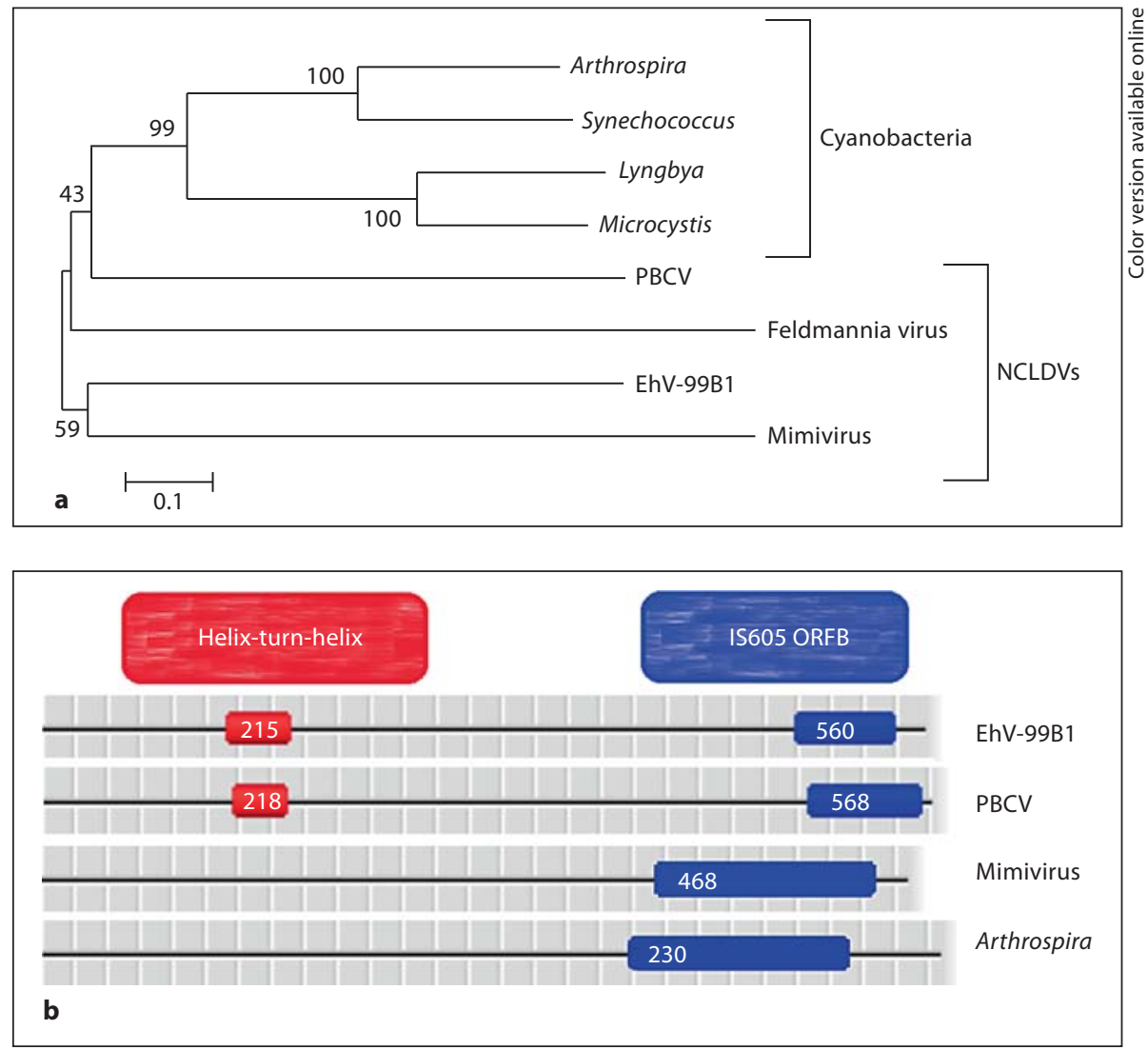

Table 4. Top BLAST hits for the putative transposase gene (ehv129A) present in the Norwegian EhV strains

\begin{tabular}{lllll}
\hline Organism & Length & Identity, \% & e value & UniProt accession \\
\hline Arthrospira platensis NIES-39 & 444 & 27.0 & $2.0 \mathrm{E}-28$ & D4ZZT1 \\
Paramecium bursaria chlorella virus AR158 & 548 & 26.0 & $8.0 \mathrm{E}-28$ & A7RAM2 \\
Lyngbya majuscula 3L & 528 & 23.0 & $3.0 \mathrm{E}-25$ & F4XNN2 \\
Microcystis aeruginosa PCC 7806 & 528 & 24.0 & $3.0 \mathrm{E}-23$ & A8YJK5 \\
Synechococcus sp. [strain JA-2-3B'a(2-13)] & 426 & 26.0 & $3.0 \mathrm{E}-23$ & Q2JQ93 \\
Acanthamoeba polyphaga mimivirus & 545 & 25.0 & $2.0 \mathrm{E}-22$ & Q5UPI0 \\
Paramecium bursaria chlorella virus NY2A & 645 & 24.0 & $5.0 \mathrm{E}-22$ & A7IWQ3 \\
Feldmannia species virus & 408 & 26.0 & $3.0 \mathrm{E}-20$ & B5LW91 \\
\hline
\end{tabular}

All the hits refer to transposase annotated sequences, and include different cyanobacteria and large DNA viruses. These results were obtained by blasting the ehv129A amino acid sequence from EhV-99B1 against the UniProt database.

genetic segregation between the EhVs from these two regions: either resulting from direct geophysical segregation or due to strong selection pressure exerted by the local host communities. This adds to the idea that the wide geographical range of this virus is probably associ- ated with some degree of geographical segregation leading to locally specific genetic traits. The identification of selfish genetic elements associated with this process provides an insight into the underlying mechanisms and factors that drive the evolution of oceanic viruses and their 
hosts (either directly or indirectly). Whilst we often marvel at the diverse end products of evolution, often the fundamental mechanisms and drivers behind the generation of such diversity remain poorly understood; here, the coccolithoviruses have presented us with a glimpse of evolution in action and the driving force behind it.

\section{Acknowledgements}

The authors would like to acknowledge support from the Norwegian Research Council (through projects HAPTODIV/No. 190307 and VIPMAP/No. 186142), the European Research Council (through project MINOS, No. 250254), and NERC (UK; through the Oceans 2025 program).

\section{References}

1 Westbroek P, Young JR, Linchooten KJ: Coccolith production (biomineralization) in the marine algae Emiliania huxleyi. J Protozool 1989;36:368-373.

2 Wilson WH, Tarran G, Zubkov MV: Virus dynamics in a coccolithophore-dominated bloom in the North Sea. Deep Sea Res Part II Top Stud Oceanogr 2002;49:2951-2963.

-3 Brussaard CP: Viral control of phytoplankton populations - a review. J Eukaryot Microbiol 2004;51:125-138.

4 Villarreal LP, Witzany G: Viruses are essential agents within the roots and stem of the tree of life. J Theor Biol 2010;262:698-710.

5 Allen MJ, Schroeder DC, Holden MTG, Wilson WH: Evolutionary history of the coccolithoviridae. Mol Biol Evo 2006;23:86-92.

-6 Wilson WH, Schroeder DC, Allen MJ, Holden MTG, Parkhill J, Barrell BG, Churcher C, Harnlin N, Mungall K, Norbertczak H, Quail MA, Price C, Rabbinowitsch E, Walker D, Craigon M, Roy D, Ghazal P: Complete genome sequence and lytic phase transcription profile of a coccolithovirus. Science 2005;309:1090-1092.

-7 Nissimov JI, Worthy CA, Rooks P, Napier JA, Kimmance SA, Henn MR, Ogata H, Allen MJ: Draft genome sequence of the coccolithovirus Emiliania huxleyi virus 203. J Virol 2011;85:13468-13469.

8 Nissimov JI, Worthy CA, Rooks P, Napier JA, Kimmance SA, Henn MR, Ogata H, Allen MJ: Draft genome sequence of the coccolithovirus ehv-84. SIGS 2011;5:1-11. $\checkmark 9$ Nissimov JI, Worthy CA, Rooks P, Napier JA, Kimmance SA, Henn MR, Ogata H, Allen MJ: Draft genome sequence of the coccolithovirus Emiliania huxleyi virus 202. J Virol 2012;86:2380-2381.

10 Nissimov JI, Worthy CA, Rooks P, Napier JA, Kimmance SA, Henn MR, Ogata H, Allen MJ: Draft genome sequence of four coccolithoviruses: Emiliania huxleyi virus EhV88, EhV-201, EhV-207, and EhV-208. J Virol 2012;86:2896-2897.

11 Allen MJ, Schroeder DC, Donkin A, Crawfurd KJ, Wilson WH: Genome comparison of two coccolithoviruses. Virol J 2006;3:15.

12 Schroeder DC, Oke J, Malin G, Wilson WH: Coccolithovirus (phycodnaviridae): characterisation of a new large dsDNA algal virus that infects Emiliania huxleyi. Arch Virol 2002;147:1685-1698.

13 Iyer LM, Balaji S, Koonin EV, Aravind L: Evolutionary genomics of nucleo-cytoplasmic large DNA viruses. Virus Res 2006;117: 156-184.

14 Raoult D, Audic S, Robert C, Abergel C, Renesto P, Ogata H, La Scola B, Suzan M, Claverie JM: The 1.2-megabase genome sequence of mimivirus. Science 2004;306: 1344-1350.

15 Sullivan MB, Huang KH, Ignacio-Espinoza JC, Berlin AM, Kelly L, Weigele PR, DeFrancesco AS, Kern SE, Thompson LR, Young S, Yandava C, Fu R, Krastins B, Chase M, Sarracino D, Osburne MS, Henn MR, Chisholm SW: Genomic analysis of oceanic cyanobacterial myoviruses compared with T4-like myoviruses from diverse hosts and environments. Environ Microbiol 2010;12:30353056.
16 Ikemura T: Codon usage and transfer-RNA content in unicellular and multicellular organisms. Mol Biol Evol 1985;2:13-34.

17 Varenne S, Buc J, Lloubes R, Lazdunski C: Translation is a non-uniform process - effect of tRNA availability on the rate of elongation of nascent polypeptide-chains. J Mol Biol 1984;180:549-576.

18 Enav H, Beja O, Mandel-Gutfreund Y: Cyanophage trnas may have a role in cross-infectivity of oceanic prochlorococcus and synechococcus hosts. ISME J 2012;6:619628.

19 Zeng QL, Chisholm SW: Marine viruses exploit their host's two-component regulatory system in response to resource limitation. Curr Biol 2012;22:124-128.

20 Thingstad TF, Skjoldal EF, Bohne RA: Phosphorus cycling and algal-bacterial competition in Sandsfjord, western Norway. Mar Ecol Prog Ser 1993;99:239-259.

21 Forterre P: The origin of viruses and their possible roles in major evolutionary transitions. Virus Res 2006;117:5-16.

22 Villarreal LP: Viruses and the Evolution of Life. Washington, American Society for Microbiology Press, 2005.

23 Allen MJ, Lanzén A, Bratbak G: Characterisation of the coccolithovirus intein. Mar Genomics 2011;4:1-7.

24 Castberg T, Thyrhaug R, Larsen A, Sandaa RA, Heldal M, van Etten JL, Bratbak G: Isolation and characterization of a virus that infects Emiliania huxleyi (Haptophyta). J Phycol 2002;38:767-774. 